\section{$\mathbf{m} / \mathbf{S}$}

médecine/sciences $1996 ; 12: 143-5$

\title{
LES MOLÉCULES DU DÉVELOPPEMENT
}

\section{Jacques Drouin}

\author{
ADRESSE \\ J. Drouin : directeur du laboratoire de génétique \\ moléculaire à l'Institut de recherches cliniques de \\ Montréal, professeur au département de biochimie \\ de l'Université de Montréal et professeur associé au \\ département de biochimie et à la division de méde- \\ cine expérimentale de l'Université McGill. Institut \\ de recherches cliniques de Montréal, 110, \\ avenue des Pins Ouest, Montréal, Québec, \\ H2W 1R7 Canada.
}

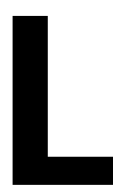

a biologie du développement est entrée dans une phase de "développement accéléré " depuis l'émergence de l'approche moléculaire. En effet, comme on l'a vu dans le domaine de l'expression génétique, la possibilité de disséquer les mécanismes au niveau moléculaire accélère grandement la vérification expérimentale des concepts et des idées. Comme dans d'autres domaines, l'approche moléculaire a été introduite dans la foulée de l'étude génétique classique. Et dans le cas de la biologie du développement, les succès remarquables de l'étude génétique viennent d'être reconnus par l'attribution des prix Nobel de médecine en 1995 à Ed Lewis, Christiane Nüesslein-Volhard et Eric Wieschaus pour leurs travaux qui ont mené à l'identification des gènes homéotiques [1].

Développement et évolution ont été depuis longtemps des sujets qui suscitent une approche globale et structuraliste, et cela parfois en opposition à une approche réductionniste qui repose sur la recherche des relations de cause à effet. Il est amusant de noter comment l'approche moléculaire réductionniste permet maintenant de trancher entre différents modèles de développement proposé au tournant du siècle (et même plus récemment) à partir d'approches structuralistes qui se voulaient globales mais qui trouvaient difficilement des assises expérimentales. Ain- si, en plus d'offrir une réévaluation de vieux concepts, l'identification des molécules régulatrices du développement nous révèle, d'une part, une conservation étonnante entre les espèces et, d'autre part, une parcimonie tout aussi surprenante puisque les mêmes molécules régulatrices sont utilisées à plusieurs niveaux du développement, qu'il s'agisse de facteurs de transcription, de facteurs de croissance, d'hormones ou des récepteurs de ces ligands.

La transgénèse et l'inactivation génique par recombinaison homologue sont dérivées de l'étude du développement précoce mais leur application touche maintenant tous les domaines de la biologie et de la médecine, bien au-delà de la seule étude du développement. Ainsi, des fonctions physiologiques complexes sont maintenant disséquées et comprises par l'analyse in vivo d'un gène ou d'un groupe de gènes. Par exemple, les fondements de la fonction cérébrale demeurent une boîte noire mais il est évident que c'est l'identification des gènes et des mécanismes du développement du cerveau qui nous permettra de comprendre le fonctionnement de cet organe complexe. Plusieurs articles de ce numéro de médecine/sciences font le point sur des acquisitions récentes en biologie du développement et, en ce début d'année, j'utiliserai cet éditorial pour faire un tour d'horizon des découvertes récentes 
du domaine et pour envisager ce que nous réserve l'année à venir.

\section{Détermination}

\section{des axes chez l'embryon}

Chez les vertébrés, les membres ont depuis longtemps constitué un modèle simple pour formuler des hypothèses sur les mécanismes du développement, en particulier sur la mise en place des axes antéro-postérieur, dorso-ventral et proximo-distal. Après avoir suggéré l'importance de gradients morphogénétiques, l'étude des structures telle la zone d'activité polarisatrice (ZPA) ou le bourrelet apical ectodermique (AER) a conduit à l'identification de plusieurs molécules régulatrices au cours des cinq dernières années. Ainsi, des molécules de différentes familles semblent impliquées dans la détermination des trois axes du développement dans les membres du poulet, dont un membre de la famille Wnt (Wnt7a), un membre de la famille "fibroblast growth factor" (FGF4) et Sonic hedgehog [2]. Ces trois molécules régulatrices sont produites dans les régions dorsale, apicale et postérieure respectivement et, bien qu'elles définissent chacune un axe, c'est par leurs interactions au niveau des tissus cibles qu'elles semblent orienter la morphogenèse $\mathrm{du}$ membre [3]. Denis Duboule résume ces découvertes et illustre de quelle façon elles ont permis de réévaluer de vieilles questions sur l'évolution des espèces (voir dans ce numéro [4]). Mais la découverte de ces molécules régulatrices a ouvert des horizons qui dépassent largement les membres. En effet, les mêmes molécules sont utilisées pour définir les axes à plus d'un niveau. Le gène sonic hedgehog (shh) en est vraisemblablement le meilleur exemple [2] : isolé au départ grâce à son homologie avec le gène de drosophile hedge$h o g$, l'importance de sa fonction est d'abord révélée par son expression et par son activité dans la zone d'activité polarisatrice des membres. Par la suite, il est montré que shh détermine, par son expression dans la notocorde, l'aspect ventral du système nerveux. Ainsi, un gradient de Shh férentielle des cellules de la plaque basale (floor plate) ou des neurones moteurs [5, 6], et l'action à distance de Shh aurait pour but l'induction du sclérotome [7]. Il ne faudrait pas conclure de cette description que Shh fait tout lors de l'établissement de la polarité dorso-ventrale : plusieurs signaux sont impliqués. Cette constatation s'impose avec force lorsque l'on considère les embryons de souris Hnf-3 $\beta-/-$ : ces embryons n'ont pas de notocorde ou de plaque basale et n'y expriment donc pas shh $\left(\mathrm{m} / \mathrm{s} n^{\circ} 1\right.$, vol. 11, p. 134) [8]. Bien que le tube neural de ces embryons soit perturbé, il conserve toujours certaines caractéristiques de l'axe dorso-ventral! Différents aspects de l'induction neurale sont discutés dans l'article de Marie-Odile Ott de ce numéro [9].

Mais plus encore, il a été observé au cours de la dernière année que Shh et d'autres molécules telle l'activine et l'un de ses récepteurs contribuent très tôt, lors de la gastrulation, à différencier le côté droit du côté gauche de l'organisme $[10,11]$. Ces molécules sont les premières à être impliquées dans cette asymétrie qui semble se mettre en place par la répression de Shh par l'activine du côté droit du nœud. Il est vraisemblable qu'il y ait d'autres régulateurs de cette asymétrie, autant en amont qu'en aval, et il sera maintenant plus aisé de « remonter " ces cascades de régulation puisque nous disposons de marqueurs moléculaires de l'asymétrie.

\section{Phylogénie et modèles de développement}

Quoi qu'en disent les tenants des concepts globaux face aux réductionnistes, il n'en demeure pas moins que les molécules régulatrices du développement constituent, de par leur conservation structurale et fonctionnelle, les meilleurs arguments appuyant des concepts d'ensemble. Ainsi, l'idée de Geoffroy St-Hilaire que les vertébrés et les arthropodes auraient un plan de développement commun bien que leurs axes dorsoventraux soient inversés, a repris de la crédibilité cet été lorsque il a été montré que des gènes homologues de la drosophile et de Xénope agis- sent autant sur des embryons homologues qu'hétérologues mais avec des effets inverses [12, 13]. En effet, tant le gène decapentaplegic $(d p p)$ de drosophile que le gène bone morphogenetic protein-4 (bmp-4) de xénope induisent un pôle dorsal chez la mouche alors qu'ils induisent des structures ventrales chez le xénope. De surcroît, les gènes short gastrulation (sog, drosophile) et chordin (chd, Xénope) ont les effets opposés dans chaque espèce. Ces études permettent donc d'envisager un plan ancestral du développement autour duquel des mécanismes souvent conservés mais interprétés différemment selon les ordres ou les espèces permettent d'engendrer des organismes aussi disparates que la drosophile ou le xénope [14].

Il est significatif à cet égard que l'inactivation de certains gènes à boîte homéo de la souris dont Hoxa-2, Mhox et $D l x-2$ produise des transformations squelettiques qui rappellent les structures équivalentes des reptiles [15-17]. Par ailleurs, même si les molécules sont conservées, il n'est pas toujours aisé de transposer leur rôle ou le plan qu'elles exécutent d'une espèce à l'autre. Ainsi, il n'est pas immédiatement évident de mettre à profit chez les vertébrés l'élucidation de signaux de la drosophile qui mettent en place les molécules des pôles antérieur et postérieur, soit les ARNm de bicoid et oskar. Ces signaux donnent lieu à des échanges d'information positionnelle entre le noyau de l'ovocyte et les cellules folliculaires qui l'entourent [18, 19]. Le site initial de ces échanges deviendra le pôle postérieur et l'échange d'information tout au long de la migration du noyau marque la région qui deviendra dorsale. Il y a donc peu en commun ici avec l'induction du mésoderme qui définit le pôle postérieur chez l'embryon de mammifère : l'avenir permettra-t-il un rapprochement entre les mécanismes sous-jacents ou s'agit-il de mécanismes totalement différents ?

\section{Le développement de la tête}

A l'autre extrémité de l'embryon, au niveau de la tête, l'étude génétique de la drosophile a encore une fois fourni l'outil, un gène, pour aborder 
un problème encore peu compris chez les mammifères, le développement de la tête et du cerveau. Le gène orthodenticle (otd) a été utilisé pour identifier des homologues chez la souris, Otx1 et Otx2. L'expression de ces gènes apparentés à bicoid est restreinte au cerveau et l'inactivation du gène $O t \times 2$ produit des souris sans tête $[20,21]$. Otx2 est donc un gène essentiel au développement de la tête. Cette démonstration étonnante de l'existence de mécanismes particuliers pour le développement de la tête confirme la notion que les gènes homéotiques du complexe Hox ne seraient pas responsables du plan du corps au-delà des oreilles [22]. Les embryons Otx2-/- ressemblent donc à ceux obtenus par l'inactivation d'un autre gène, lim-1 $\left(\mathrm{m} / \mathrm{s} n^{\circ} 5\right.$, vol. 11, p. 783) [23] : l'un de ces gènes mérite-t-il plus que l'autre le titre «d'organisateur de la tête » ou font-ils tous deux partie du même plan intégré ? Quoi qu'il en soit, il semble que la boîte noire que constitue le développement du cerveau soit ouverte et que nous en comprendrons bientôt les mécanismes. Et il est aussi probable que cela jettera une nouvelle lumière sur l'étude de la fonction cérébrale. Il y a loin de la tête de mouche au cerveau humain, mais peut-être moins qu'il n'y paraît!

\section{TIRÉS À PART}

J. Drouin.

\section{RÉFÉRENCES}

1. Deutsch J, Lamourisnard C, Lepesant JA. Le prix Nobel 95 attribué à Ed Lewis, Christiane Nüesslein-Volhard et Eric Wieschaus : la reconnaissance de la génétique du développement. médecine/sciences $1995 ; 11$ : 1625-8.

2. Concordet J. Morphogenèse, acide rétinoïque... et Sonic Hedgehog. médecine/sciences $1994 ; 10: 570-3$.

3. Yang Y, Niswander L. Interaction between the signaling molecules WNT7a and SHH during vertebrate limb development : dorsal signals regulate anteroposterior patterning. Cell 1995 ; 80 : 939-47.

4. Duboule D, Sordino P. Des nageoires aux membres : l'apport de la génétique moléculaire du développement dans l'étude de l'évolution des morphologies chez les vertébrés. médecine/sciences 1996; $12: 147-54$.

5. Roelink H, Porter JA, Chiang C, Tanabe Y, Chang DT, Beachy PA, Jessell TM. Floor plate and motor neuron induction by different concentrations of the amino-terminal cleavage product of sonic hedgehog autoproteolysis. Cell $1995 ; 81: 445-55$.

6. Marti E, Bumcrot DA, Takada R, McMahon AP. Requirement of $19 \mathrm{~K}$ form of Sonic hedgehog for induction of distinct ventral cell types in CNS explants. Nature 1995 ; 375 : 322-5.

7. Fan CM, Porter JA, Chiang C, Chang DT, Beachy PA, Tessier-Lavigne M. Long-range sclerotome induction by sonic hedgehog : direct role of the amino-terminal cleavage product and modulation by the cyclic AMP signaling pathway. Cell $1995 ; 81: 457-65$.

8. Ang SL, Rossant J. HNF-3 beta is essential for node and notochord formation in mouse development. Cell 1994; $78: 561-74$.

9. Ott MO. L'induction neurale chez la souris. médecine/sciences $1996 ; 12: 209-14$.

10. Levin M, Johnson RL, Stern CD, Kuehn M, Tabin C. A molecular pathway determining left-right asymmetry in chick embryogenesis. Cell 1995; 82 : 803-14.

11. Concordet JP. Asymétries gauche-droite chez les vertébrés. médecine/sciences 1996 $12: 207-8$.

12. Holley SA, Jackson PD, Sasai Y, Lu B, DeRobertis EM, Hoffmann FM, Ferguson EL. A conserved system for dorsal-ventral patterning in insects and vertebrates involving sog and chordin. Nature $1995 ; 376: 249-53$.
13. Schmidt J, Francois V, Bier E, Kimelman D. Drosophila short gastrulation induces an ectopic axis in Xenopus : evidence for conserved mechanisms of dorsal-ventral patterning. Development 1995 ; 121 : 431928.

14. Hogan BLM. Molecular morphogens Upside-down ideas vindicated. Nature 1995 ; $376: 210-1$.

15. Rijli FM, Mark M, Lakkaraju S, Dierich A, Dolle P, Chambon P. A homeotic transformation is generated in the rostral branchial region of the head by disruption of Hoxa-2, which acts as a selector gene. Cell $1993 ; 75$ : 1333-49.

16. Martin JF, Bradley A, Olson EN. The paired-like homeo box gene MHox is required for early events of skeletogenesis in multiple lineages. Genes Dev 1995 ; 9 : 1237-49.

17. Qiu MS, Bulfone A, Martinez S, Meneses II, Shimamura K, Pedersen RA, Rubenstein JLR. Null mutation of $D l x-2$ results in abnormal morphogenesis of proximal first and second branchial arch derivatives and abnormal differentiation in the forebrain. Genes Dev 1995 ; 9 : 2523-38.

18. Gonzalez-reyes A, Elliott H, Stjohnston D. Polarization of both major body axes in drosophila by Gurken-Torpedo signaling. Nature 1995 ; 375 : 654-8.

19. Roth S, Neumansilberberg FS, Barcelo G, Schupbach T. Cornichon and the EGF receptor signaling process are necessary for both anterior posterior and dorsal-ventral pattern formation in drosophila. Cell 1995 ; $81: 967-78$.

20. Acampora D, Mazan S, Lallemand Y, Avantaggiato V, Maury M, Simeone A, Brûlet $P$. Forebrain and midbrain regions are deleted in $\operatorname{Ot} \times 2-/-$ mutants due to a defective anterior neuroectoderm specification during gastrulation. Development 1995 ; 121 : 327990

21. Matsuo I, Kuratani S, Kimura C, Takeda $\mathrm{N}$, Aizawa S. Mouse Otx2 functions in the formation and patterning of rostral head. Genes Dev $1995 ; 9$ : 2646-58.

22. Krumlauf R. Hox genes in vertebrate development. Cell $1994 ; 78$ : 191-201.

23. Shawlot W, Behringer RR. Requirement for Lim1 in head-organizer function. Nature $1995 ; 374$ : 425-30. 